\title{
Doppler Ultrasound Study on the Effects of Different Blood Uric Acid Levels on the Carotid Artery Intima- media Thickness in Patients with Gout
}

\section{Wantai Dang}

Clinical Medical College and The First Affiliated Hospital of Chengdu Medical College Jing $\mathrm{Hu}$

Clinical Medical College and The First Affiliated Hospital of Chengdu Medical College

Hui Luo

Clinical Medical College and The First Affiliated Hospital of Chengdu Medical College

Danling Luo

Clinical Medical College and The First Affiliated Hospital of Chengdu Medical College

\section{Xiaohui Xu}

Clinical Medical College and The First Affiliated Hospital of Chengdu Medical College

\section{Jingguo Zhou}

Clinical Medical College and The First Affiliated Hospital of Chengdu Medical College

Jian Liu ( $\nabla$ liujiansh@126.com )

Clinical Medical College and The First Affiliated Hospital of Chengdu Medical College, Chengdu, Sichuan https://orcid.org/0000-0001-5148-5069

\section{Research article}

Keywords: Primary gout, Blood uric acid level, Doppler ultrasound, Common carotid artery, Resistance index

Posted Date: June 2nd, 2021

DOl: https://doi.org/10.21203/rs.3.rs-556689/v1

License: (c) (i) This work is licensed under a Creative Commons Attribution 4.0 International License. Read Full License 


\title{
Doppler ultrasound study on the effects of different blood uric acid levels on the
}

\section{carotid artery intima-media thickness in patients with gout}

\author{
Wantai Dang ${ }^{1 \dagger}$, Jing $\mathrm{Hu}^{2 \dagger}$, Hui $\mathrm{Luo}^{2}$, Danling $\mathrm{Luo}^{2}$, Xiaohui $\mathrm{Xu}^{2}$, Jingguo Zhou ${ }^{1}, \mathrm{Jian}^{\mathrm{Li}}{ }^{2} *$ \\ 1 Department of Rheumatology and Immunology, Clinical Medical College and The First \\ Affiliated Hospital of Chengdu Medical College, Chengdu, Sichuan, 610500, PR China. \\ 2 Department of Ultrasound, Clinical Medical College and The First Affiliated Hospital of \\ Chengdu Medical College, Chengdu, Sichuan, 610500, PR China. \\ *Correspondence: Jian Liu, E-mail:liujiansh@126.com \\ ${ }^{\dagger}$ These authors contributed equally as first authors.
}

\begin{abstract}
Objective: Doppler ultrasonography is used to study the effects of different serum uric acid (SUA) levels in primary gout (PG) on the carotid artery intima-media thickness (CIMT) and its risk factors and to provide clinical evidence for early prevention and treatment of systematic gout damage.
\end{abstract}

Methods: We obtained information of 237 patients with PG (intermittent period) and estimated the association between gout and common carotid artery intima-media thickness (CCAIMT), carotid plaque formation, and blood flow resistance index (RI)) using binary logistic regression.

Results: The CCAIMT of patients with PG was significantly correlated with different SUA levels, age, course of the disease, blood routine laboratory parameters etc $(P<0.05)$. The CCAIMT of patients with PG combined with the tophi, secondary hypertension, or diabetes group was significantly different from that in the uncombined group $(P<0.05)$. The age, disease course, body mass index (BMI), blood routine indexes, liver function, renal function, and lipid 
metabolism-related indexes of patients with gout combined with the carotid plaque group and without the carotid plaque group were significantly different $(P<0.05)$. Age $(\mathrm{OR}, 95 \% \mathrm{CI}=1.136$, 1.073-1.202), disease course ( $\mathrm{OR}, 95 \% \mathrm{CI}=1.236,1.084-1.410)$, and low-density lipoprotein cholesterol (LDL-C) $(\mathrm{OR}, 95 \% \mathrm{CI}=6.982,2.460-19.816)$ were risk factors for CCAIMT of more than $0.0858 \mathrm{~cm}$, whereas SUA $(\mathrm{OR}, 95 \% \mathrm{CI}=0.994,0.988-0.999)$ and Lymphocyte percentage $(\mathrm{LY} \%)(\mathrm{OR}, 95 \% \mathrm{CI}=0.842,0.723-0.980)$ were protective factors. Age was a risk factor for carotid plaque (OR, 95\% CI = 1.137, 1.071-1.207), and SUA was a protective factor (OR, 95\% CI $=0.992,0.986-0.998)$. Moreover, secondary hypertension $(\mathrm{OR}, 95 \% \mathrm{CI}=3.496,1.442-8.479)$, PLT $(\mathrm{OR}, 95 \% \mathrm{CI}=1.005,1.001-1.010)$, and SUA $(\mathrm{OR}, 95 \% \mathrm{CI}=1.003,1.000-1.005)$ were risk factors for common carotid artery RI of $\geq 0.7$ in patients with gout.

Conclusion: Age, disease course, blood routine indexes, renal function, and lipid metabolism of patients with gout with different SUA levels were closely related to carotid intima-media diseases. Age, disease course, and LDL-C were the risk factors for carotid artery intima-media lesions in patients with PG, whereas SUA and LY\% might be protective factors.

\section{Keywords}

Primary gout, Blood uric acid level, Doppler ultrasound, Common carotid artery, Resistance index

\section{Introduction}

Gout is a metabolic and inflammatory/immune disease caused by impaired purine metabolism and/or decreased uric acid excretion. The continuous increase in blood uric acid causes urate (monosodium urate, MSU) crystals to precipitate and deposit in the joints or other tissues [1,2]. The global prevalence of gout ranges from $<1 \%$ to $6.8 \%$, and the incidence is 0.58-2.89 per 1,000 people, with males having a higher prevalence than females. In addition, there 
are ethnic differences in the disease, and the prevalence increases with age [3]. MSU crystals can not only act on joints but also on multiple target organs, such as blood vessels, the heart, and the kidneys, and are closely related to atherosclerosis, hypertension, metabolic syndrome, and chronic kidney disease $[2,4,5,9]$. Presently, joint fluid or tophi tissue puncture to detect MSU crystals is the gold standard for diagnosing gouty arthritis, but this is an invasive test. The gout classification standards formulated in 2015 by the American College of Rheumatology (ACR) and the European League Against Rheumatism (EULAR) and the EULAR updated gout diagnosis recommendations in 2018 emphasize the importance of ultrasound in the diagnosis of gout [1,2]. Ultrasound detection of peripheral arteries has been widely used in clinical practice and has become an important auxiliary method for detecting atherosclerosis [6]. As a superficial artery, the carotid artery can reflect the degree of systemic atherosclerosis. Studies have shown that carotid artery intima-media thickness (CIMT) is one of the indicators that reflect the degree of carotid arteriosclerosis with high sensitivity and specificity. Two changes occur in atherosclerosis: the early change is intima-media thickening, and the late change is atherosclerotic plaque formation [15]. The common carotid artery resistive index (CCARI) is affected in the early stage of the pathological process of cardiovascular disease, which reflects disease progression. Therefore, changes in the CCARI are a key indicator of pathological changes in important vascular regions [7, 8].

Studies have shown that serum uric acid (SUA) is significantly related to carotid artery diseases [9-12], but some studies have shown that SUA is not significantly related to carotid artery diseases $[4,5,13,14]$. The aforementioned studies were mostly based on pure blood uric acid levels or studies on non-gout patients. Thus, the effects of different blood uric acid levels on CIMT in 
patients with primary gout remain unclear. This study was conducted to investigate the effects of different blood uric acid levels on CIMT in patients with primary gout using Doppler ultrasound technology and to provide clinical evidence for the diagnosis and treatment of gout system damage.

\section{Participants and methods}

\section{Participants}

In this study, 237 patients with primary gout (intermittent period) were enrolled. The inclusion criteria were as follows: patients with primary gout that met the 2015 ACR/EULAR gout diagnostic criteria [2]; patients aged 16-90 years; patients who did not receive uric acid-lowering and other related treatments within 3 months. The exclusion criteria were as follows: patients with secondary gout caused by myeloproliferative disease, tumor radiotherapy, chemotherapy, and kidney disease; patients with a history of cervical radiotherapy, carotid artery intervention or surgery, and carotid artery trauma; patients with rheumatoid arthritis, reactive arthritis, psoriatic arthritis, spondyloarthritis, or other autoimmune diseases; patients with severe heart and kidney diseases, blood infections, vasculitis, and other infectious diseases; patients with serious diseases of other systems affecting the investigation and patients who refuse to sign informed consent. This study was approved by the Medical Ethics Committee of the First Affiliated Hospital of Chengdu Medical College, and all research subjects provided informed consent.

\section{Methods}

\section{General clinical information/characteristics}

Medical history was asked by the researcher, and the patient data including gender, age, disease duration of gout, presence of tophus, secondary hypertension and diabetes mellitus were 
recorded. Height and weight were measured, and BMI was calculated. All subjects signed the informed consent.

\section{Blood routine indexes and biochemical indexes}

Fasting venous blood and clean midstream urine were collected from the patients in the morning (at least 8 hours of overnight dietary restriction). The blood routine indexes (Automatic blood analyzer: Japan Sysmex XN-9000) and biochemical indexes (Automatic biochemical analyzer: Japan Hitachi Limited 7600, Erythrocyte sedimentation rate analyzer: USA Vital Diagnostics Monitor-100, Automatic urine analyzer: Japan Sysmex UF-4000) were detected. All indexes include:

Blood routine indexes: white blood cell count (WBC), red blood cell count (RBC), hemoglobin (HGB), platelets count (PLT), neutrophil percentage (NE), lymphocyte percentage (LY\%), basophil cell percentage (BA).

Liver function indexes: alanine transaminase (ALT), aspartate transaminase (AST), total bilirubin (TBIL), direct bilirubin (DBIL).

Kidney function indexes:urea (UREA), creatinine (CREA), serum uric acid (SUA), cystatin $\mathrm{C}$ (CysC), $\beta 2$-microglobulin ( $\beta-2 \mathrm{MG})$, uric-PH (U-PH), 24h urine uric acid/L (UUA).

Lipid metabolism indexes:total protein (TP), albumin (ALB), globulin (GLB), ration of albumin to globulin (A:G), triglyceride (TG),cholesterol (TC), high density lipoprotein cholesterol (HDL-C), low density lipoprotein cholesterol (LDL-C), apolipoprotein A-I (ApoA-I), apolipoprotein B (ApoB), ApoA-I/ApoB, homocysteine (Hcy), lipoprotein (a) (Lp(a)).

Glucose metabolism indexes: fasting plasma glucose (FPG), glycated hemoglobin (HBA1C), average blood glucose (AG). 
Inflammatory indexes: C-reactive protein (CRP), erythrocyte sedimentation rate (ESR).

\section{Cervical ultrasound examination}

The PHILIPS EPIQ7c and Mindray resona8 ultrasonic imaging instrument was used for ultrasound inspection, with a linear array probe frequency of 9-12 MHz using the cervical blood vessel inspection mode. The patient underwent bilateral carotid artery ultrasound examination in the supine position under a calm breathing state. The gray-scale imaging mode was used to scan the carotid artery first by transverse section and then longitudinal section. Scanning was started from the bifurcation of the innominate artery on the right side and the beginning of the common carotid artery on the left side. The carotid artery (proximal, middle, and distal segment), internal carotid artery, and external carotid artery were continuously scanned. While avoiding plaque and clearly showing the front and rear walls, the CIMT (thickest part) was measured. If no obvious thick spot is observed by the naked eye, the intima-media thickness of the common carotid artery (CCAIMT) of 1-1.5 cm below the bifurcation of the common carotid artery was measured (Fig. 1-A), and the average of three measurements was calculated. Color Doppler flow imaging was used to observe the blood flow filling state of the aforementioned arteries. Pulse Doppler ultrasound was used to measure the peak systolic velocity, end diastolic velocity, and resistive index (RI) of the common and internal carotid arteries (Fig. 1-C). The sampling volume was fixed at $2 \mathrm{~mm}$, placed in the brightest part of the center of the blood vessel. The best sound beam blood flow angle $\left(<60^{\circ}\right)$ was set, the blood flow spectrum of three cardiac cycles was measured, and the average value was calculated. The aforementioned ultrasound detection was performed on the left and right carotid arteries, and the average values of bilateral CCAIMT, common carotid artery (RI) CCARI, and internal carotid artery resistive index (ICARI) were calculated. Plaque was defined as 
(Fig. 1-B). In this study, the average CCAIMT values of greater than $75 \%$ were regarded as abnormal, and the average CCARI and average ICARI values of $\geq 0.70$ were regarded as abnormal.
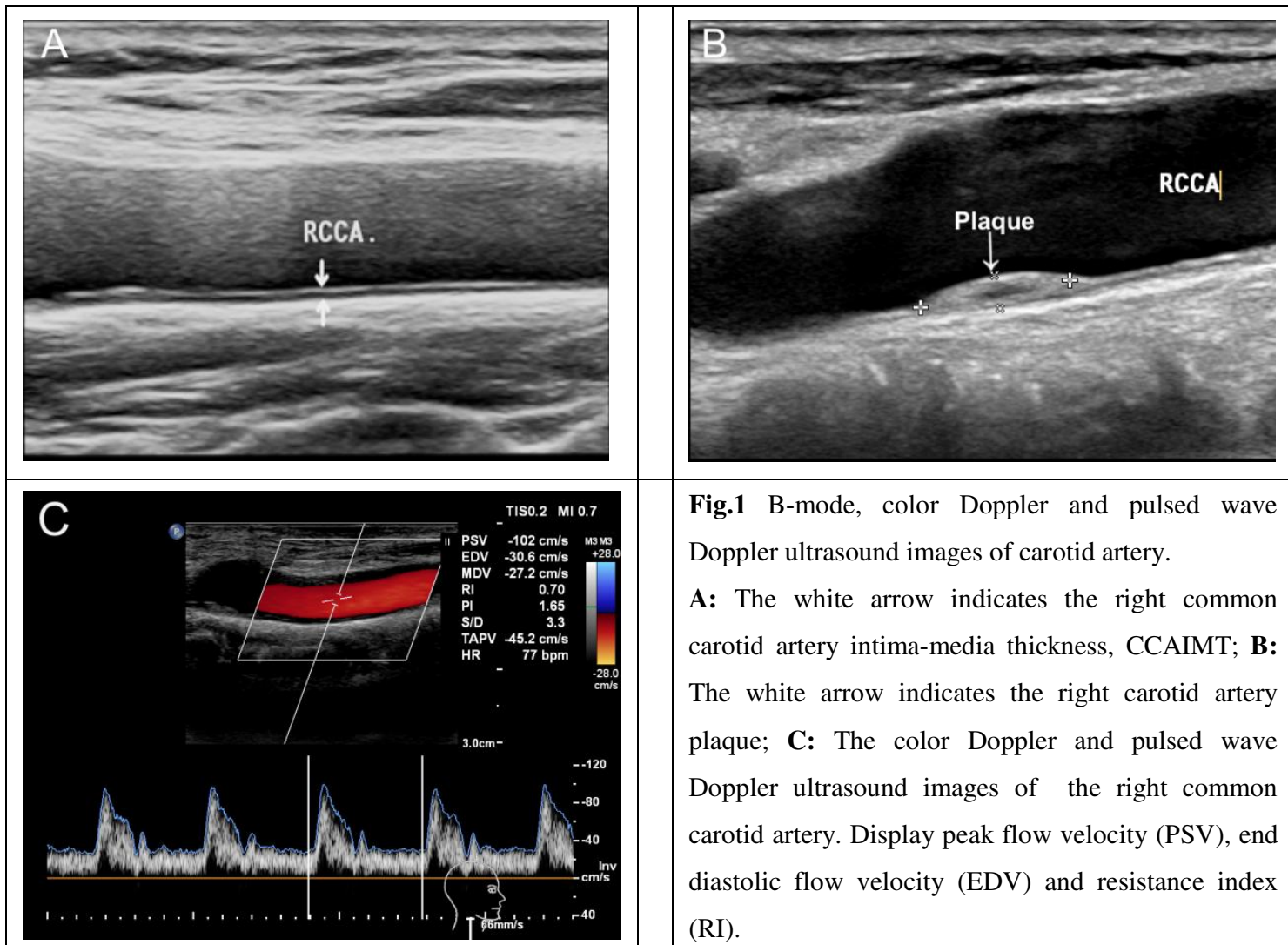

Fig.1 B-mode, color Doppler and pulsed wave Doppler ultrasound images of carotid artery.

A: The white arrow indicates the right common carotid artery intima-media thickness, CCAIMT; B: The white arrow indicates the right carotid artery plaque; C: The color Doppler and pulsed wave Doppler ultrasound images of the right common carotid artery. Display peak flow velocity (PSV), end diastolic flow velocity (EDV) and resistance index (RI).

\section{Statistical analysis}

Statistical Package for the Social Sciences version 22.0 was used for all statistical analyses. In the baseline characteristics, categorical variables were expressed as counts and percentages, and continuous variables were expressed as mean \pm standard deviation or quartiles. The Mann-Whitney rank sum test was used to evaluate the differences between two independent samples, spearman correlation analysis was used to test the correlation between different parameters, and binary logistic regression analysis was used to evaluate the risk factors of carotid artery diseases. $P$-values of less than 0.05 were used to indicate that the difference is statistically 
significant.

\section{Results}

\section{Baseline characteristics}

Among the 237 patients enrolled, 224 were male (94.5\%) and 13 were female (5.5\%). The average age of the patients was $46.350 \pm 15.298$ years, the average course of the disease was $4.729 \pm 5.441$ years, the average height of the patients was $167.270 \pm 6.888 \mathrm{~cm}$, the average weight was $73.310 \pm 12.599 \mathrm{~kg}$, and the average BMI was $26.163 \pm 3.814 \mathrm{~kg} / \mathrm{m}^{2}$. Among them, 44 had secondary hypertension, and 193 had no hypertension. In addition, 33 had secondary diabetes, and 204 had no diabetes. All patients underwent joint ultrasound examination, 117 of whom patients had tophi, whereas 120 patients had no tophi (Table 1). All patients underwent cervical vascular ultrasound examination. The specific ultrasound indices are shown in Table 2.

Table1. Clinical characteristics of patients with gout

\begin{tabular}{|c|c|c|c|}
\hline Characteristics & & $\mathrm{N}$ & mean $\pm \mathrm{SD}$ \\
\hline Male (n, \%) & & $224(94.5 \%)$ & \\
\hline Female (n, \%) & & $13(5.5 \%)$ & \\
\hline Age (year) & & 237 & $46.350 \pm 15.298$ \\
\hline disease duration (year) & & 237 & $4.729 \pm 5.441$ \\
\hline High $(\mathrm{cm})$ & & 237 & $167.270 \pm 6.888$ \\
\hline Weight (kg) & & 237 & $73.310 \pm 12.599$ \\
\hline BMI & & 237 & $26.163 \pm 3.814$ \\
\hline \multirow[t]{2}{*}{ Hypertension (n, \%) } & No & $193(81.4 \%)$ & \\
\hline & Yes & $44(18.6 \%)$ & \\
\hline \multirow[t]{2}{*}{ Diabetes Mellitus (n, \%) } & No & $204(86.1 \%)$ & \\
\hline & Yes & $33(13.9 \%)$ & \\
\hline \multirow[t]{2}{*}{ Tophi (n, \%) } & No & $120(50.6 \%)$ & \\
\hline & Yes & $117(49.4 \%)$ & \\
\hline
\end{tabular}


Table2. Carotid ultrasonography characteristics of patients with gout

\begin{tabular}{lcccccc}
\hline Characteristics & & $\mathrm{N}$ & mean $\pm \mathrm{SD}$ & $\mathrm{P}_{25}$ & $\mathrm{P}_{50}$ & $\mathrm{P}_{75}$ \\
\hline Plaque (n, \%) & No & $155(65.4 \%)$ & & & & \\
& Yes & $82(34.6 \%)$ & & & & \\
Mean & & & & & & \\
CCAIMT(cm) & & & & & & \\
Mean CCARI & & 237 & $0.077 \pm 0.018$ & 0.0650 & 0.0740 & 0.0858 \\
Mean ICARI & & 237 & $0.596 \pm 0.049$ & 0.675 & 0.705 & 0.740 \\
\hline
\end{tabular}

\section{Correlation analysis}

2.1 Analysis of the correlation between SUA and general clinical characteristics and various laboratory indicators

In the gout patients, SUA level was positively correlated with RBC, HGB, BA, ALT, CREA, CysC, UUA, TP, ALB, TG, ApoB $(P<0.05)$, and negatively correlated with age, U-pH, FPG, ESR $(P<0.05)$. (Table 3)

Table 3. Correlation between serum uric acid and general clinical characteristics and laboratory indicators

\begin{tabular}{lcc}
\hline & \multicolumn{2}{c}{$\mathrm{SUA}(\mu \mathrm{mol} / \mathrm{L})$} \\
\cline { 2 - 3 } Age $($ year $)$ & -0.162 & 0.014 \\
RBC $\left(10^{12} / \mathrm{L}\right)$ & 0.177 & 0.007 \\
HGB $(\mathrm{g} / \mathrm{L})$ & 0.195 & 0.003 \\
BA $(\%)$ & 0.143 & 0.031 \\
ALT $(\mathrm{U} / \mathrm{L})$ & 0.156 & 0.019 \\
CREA $(\mu \mathrm{mol} / \mathrm{L})$ & 0.281 & $<0.001$ \\
CysC $(\mathrm{mg} / \mathrm{L})$ & 0.141 & 0.032 \\
U-PH & -0.183 & 0.007 \\
UUA $(\mu \mathrm{mol} / \mathrm{L})$ & 0.169 & 0.015 \\
TP $(\mathrm{g} / \mathrm{L})$ & 0.203 & 0.002 \\
ALB $(\mathrm{g} / \mathrm{L})$ & 0.175 & 0.008 \\
TG $(\mathrm{mmol} / \mathrm{L})$ & 0.241 & $<0.001$ \\
ApoB $(\mathrm{g} / \mathrm{L})$ & 0.164 & 0.019 \\
FPG $(\mathrm{mmol} / \mathrm{L})$ & -0.245 & 0.001 \\
ESR $(\mathrm{mm} / \mathrm{h})$ & -0.138 & 0.046 \\
\hline
\end{tabular}

2.2 Correlation analysis between carotid ultrasound parameters and general clinical features and laboratory indicators 
In the gout patients, CCAIMT was positively correlated with age, disease duration of gout, NE, UREA, CysC, $\beta 2-M G$, CRP, ESR $(P<0.05)$, and negatively correlated with RBC, HGB, PLT, LY\%, ALT, TBIL, DBIL, SUA, TP, ALB $(P<0.05)$. (Table 4)

The CCAIMT of the patients with gout in the secondary hypertension group was greater than that of the patients with gout in the non-secondary hypertension group; the CCAIMT of the patients with gout in the secondary diabetes group was greater than that of the patients with gout in the non-secondary diabetes group; and the CCAIMT of the patients in the combined tophi group was greater than that of the patients in the non-combined tophi group. The aforementioned differences were statistically significant $(P<0.05)$ (Fig. 2).

Table 4. Correlation between CCAIMT、CCARI、ICARI and general clinical characteristics and laboratory indicators

\begin{tabular}{|c|c|c|c|c|c|}
\hline \multicolumn{3}{|c|}{ Mean CCA-IMT (cm) } & & \multicolumn{2}{|c|}{ Mean CCARI } \\
\hline & $r$ & $P$ & & $r$ & $P$ \\
\hline Age (year) & 0.651 & $<0.001$ & $\operatorname{PLT}\left(10^{9} / \mathrm{L}\right)$ & 0.156 & 0.018 \\
\hline Disease duration (year) & 0.345 & $<0.001$ & TBIL $(\mu \mathrm{mol} / \mathrm{L})$ & 0.132 & 0.046 \\
\hline $\mathrm{RBC}\left(10^{12} / \mathrm{L}\right)$ & -0.442 & $<0.001$ & DBIL $(\mu \mathrm{mol} / \mathrm{L})$ & 0.150 & 0.024 \\
\hline $\operatorname{HGB}(\mathrm{g} / \mathrm{L})$ & -0.444 & $<0.001$ & SUA $(\mu \mathrm{mol} / \mathrm{L})$ & 0.166 & 0.011 \\
\hline $\operatorname{PLT}\left(10^{9} / \mathrm{L}\right)$ & -0.151 & 0.022 & & & \\
\hline $\mathrm{NE}(\%)$ & 0.237 & $<0.001$ & & & \\
\hline LY (\%) & -0.287 & $<0.001$ & & \multicolumn{2}{|c|}{ Mean ICARI } \\
\hline ALT (U/L) & -0.144 & 0.030 & & $r$ & $P$ \\
\hline TBIL ( $\mu \mathrm{mol} / \mathrm{L})$ & -0.199 & 0.002 & Age (year) & 0.154 & 0.018 \\
\hline DBIL ( $\mu \mathrm{mol} / \mathrm{L})$ & -0.164 & 0.013 & $\mathrm{WBC}\left(10^{9} / \mathrm{L}\right)$ & -0.132 & 0.046 \\
\hline UREA $(\mathrm{mmol} / \mathrm{L})$ & 0.253 & $<0.001$ & $\operatorname{DBIL}(\mu \mathrm{mol} / \mathrm{L})$ & 0.200 & 0.002 \\
\hline SUA $(\mu \mathrm{mol} / \mathrm{L})$ & -0.156 & 0.018 & $\mathrm{TC}(\mathrm{mmol} / \mathrm{L})$ & -0.163 & 0.019 \\
\hline CysC (mg/L) & 0.335 & $<0.001$ & $\mathrm{LDL}-\mathrm{C}(\mathrm{mmol} / \mathrm{L})$ & -0.155 & 0.026 \\
\hline$\beta 2-\mathrm{MG}(\mathrm{mg} / \mathrm{L})$ & 0.407 & $<0.001$ & ApoB (g/L) & -0.148 & 0.035 \\
\hline $\mathrm{TP}(\mathrm{g} / \mathrm{L})$ & -0.305 & $<0.001$ & & & \\
\hline $\operatorname{ALB}(g / L)$ & -0.278 & $<0.001$ & & & \\
\hline CRP (mg/L) & 0.245 & $<0.001$ & & & \\
\hline $\operatorname{ESR}(\mathrm{mm} / \mathrm{h})$ & 0.318 & $<0.001$ & & & \\
\hline
\end{tabular}




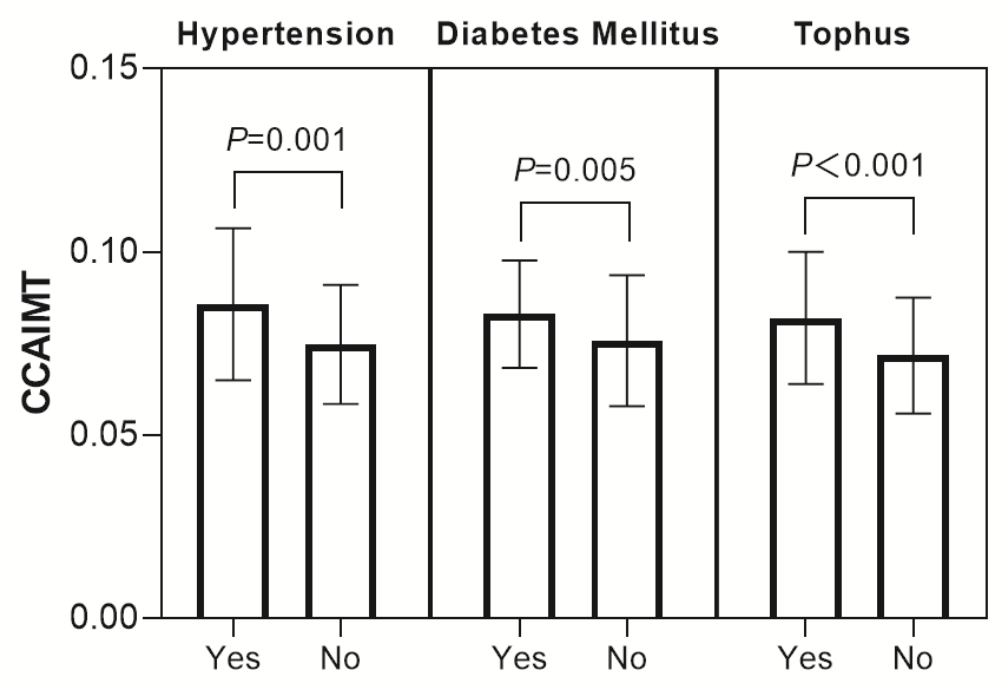

Fig. 2: CCAIMT of gout patients with secondary hypertension or diabetes mellitus or tophi is greater than that of the uncombined group $(P<0.05)$.

All gout patients were divided into Q1-Q4 groups according to the four quartiles of their SUA level: Q1 SUA $\leq 437 \mu \mathrm{mol} / \mathrm{L}, \mathrm{Q} 2437<\mathrm{SUA} \leq 510 \mu \mathrm{mol} / \mathrm{L}, \mathrm{Q} 3510<\mathrm{SUA} \leq 605 \mu \mathrm{mol} / \mathrm{L}$, and Q4 SUA $>605 \mu \mathrm{mol} / \mathrm{L}$.

In Q1 group (SUA $\leq 437 \mu \mathrm{mol} / \mathrm{L}$ ), CCAIMT was positively correlated with age, disease duration, BA, CysC, CRP,ESR, but negatively correlated with RBC, ALT, TP, ALB $(P<0.05)$.In Q2 group $(437<\mathrm{SUA} \leq 510 \mu \mathrm{mol} / \mathrm{L})$, CCAIMT was positively correlated with age, disease duration, UREA, CysC, $\beta 2-\mathrm{MG}$, but negatively correlated with RBC, HGB, TP $(P<0.05)$.In Q3 group $(510<\mathrm{SUA} \leq 605 \mu \mathrm{mol} / \mathrm{L})$, CCAIMT was positively correlated with age, disease duration, NE, UREA, CREA, CysC, $\beta 2-\mathrm{MG}, \mathrm{Hcy}$, CRP, ESR, but negatively correlated with RBC, HGB, LY\%, TBil, U-PH, ALB $(P<0.05)$.In Q4 group $(\mathrm{SUA}>605 \mu \mathrm{mol} / \mathrm{L})$, CCAIMT was positively correlated with age, disease duration, CREA, CysC, $\beta 2-\mathrm{MG}$, FPG, but negatively correlated with RBC, HGB, TP $(P<0.05)$. (Table 5) 
Table 5.Correlation between CCAIMT and general clinical characteristics and laboratory indicators in different groups

\begin{tabular}{llll}
\multicolumn{4}{c}{ Mean CCA-IMT $(\mathrm{cm})$} \\
\hline Q1 & Q2 & Q3 & Q4 \\
$(\mathrm{SUA} \leq 437 \mu \mathrm{mol} / \mathrm{L})$ & $(437<\mathrm{SUA} \leq 510 \mu \mathrm{mo}$ & $(510<\mathrm{SUA} \leq 605 \mu \mathrm{mo}$ & $(\mathrm{SUA}>605 \mu \mathrm{mol} / \mathrm{L})$ \\
& $1 / \mathrm{L})$ & $1 / \mathrm{L})$ &
\end{tabular}

\begin{tabular}{|c|c|c|c|c|c|c|c|c|}
\hline & $r$ & $P$ & $r$ & $P$ & $r$ & $P$ & $r$ & $P$ \\
\hline Age (year) & 0.585 & $<0.001$ & 0.675 & $<0.001$ & 0.746 & $<0.001$ & 0.548 & $<0.001$ \\
\hline $\begin{array}{l}\text { disease } \\
\text { duration(year) }\end{array}$ & 0.316 & 0.016 & 0.291 & 0.027 & 0.610 & $<0.001$ & 0.398 & 0.003 \\
\hline High (cm) & -0.303 & 0.021 & -0.366 & 0.005 & -0.253 & 0.051 & -0.136 & 0.322 \\
\hline $\operatorname{RBC}\left(10^{12} / \mathrm{L}\right)$ & -0.277 & 0.037 & -0.417 & 0.001 & -0.523 & $<0.001$ & -0.415 & 0.002 \\
\hline HGB (g/L) & -0.246 & 0.065 & -0.470 & $<0.001$ & -0.534 & $<0.001$ & -0.381 & 0.004 \\
\hline NE (\%) & 0.177 & 0.187 & 0.153 & 0.260 & 0.415 & 0.001 & 0.147 & 0.286 \\
\hline LY (\%) & -0.197 & 0.143 & -0.222 & 0.100 & -0.492 & $<0.001$ & -0.161 & 0.241 \\
\hline BA $(\%)$ & 0.264 & 0.047 & -0.053 & 0.700 & -0.089 & 0.498 & -0.211 & 0.122 \\
\hline ALT (U/L) & -0.262 & 0.049 & 0.041 & 0.762 & -0.079 & 0.547 & -0.152 & 0.266 \\
\hline TBIL $(\mu \mathrm{mol} / \mathrm{L})$ & -0.122 & 0.365 & -0.186 & 0.166 & -0.295 & 0.022 & -0.193 & 0.157 \\
\hline UREA (mmol/L) & 0.106 & 0.427 & 0.411 & 0.001 & 0.397 & 0.002 & 0.213 & 0.119 \\
\hline CREA $(\mu \mathrm{mol} / \mathrm{L})$ & -0.019 & 0.889 & 0.004 & 0.977 & 0.286 & 0.027 & 0.280 & 0.038 \\
\hline CysC (mg/L) & 0.259 & 0.049 & 0.297 & 0.024 & 0.577 & $<0.001$ & 0.298 & 0.028 \\
\hline$\beta 2-\mathrm{MG}(\mathrm{mg} / \mathrm{L})$ & 0.238 & 0.075 & 0.419 & 0.001 & 0.591 & $<0.001$ & 0.452 & 0.001 \\
\hline U-PH & -0.149 & 0.283 & -0.184 & 0.186 & -0.295 & 0.027 & 0.082 & 0.553 \\
\hline TP $(\mathrm{g} / \mathrm{L})$ & -0.344 & 0.009 & -0.276 & 0.037 & -0.237 & 0.069 & -0.269 & 0.047 \\
\hline ALB (g/L) & -0.375 & 0.004 & -0.236 & 0.077 & -0.348 & 0.006 & -0.101 & 0.463 \\
\hline $\mathrm{Hcy}(\mu \mathrm{mol} / \mathrm{L})$ & -0.073 & 0.613 & 0.047 & 0.749 & 0.277 & 0.045 & 0.237 & 0.105 \\
\hline CRP (mg/L) & 0.329 & 0.015 & 0.222 & 0.109 & 0.313 & 0.018 & 0.117 & 0.410 \\
\hline ESR $(\mathrm{mm} / \mathrm{h})$ & 0.367 & 0.006 & 0.189 & 0.179 & 0.401 & 0.003 & 0.248 & 0.090 \\
\hline FPG (mmol/L) & -0.139 & 0.362 & 0.110 & 0.456 & 0.068 & 0.641 & 0.290 & 0.045 \\
\hline
\end{tabular}

Statistically significant differences in the general clinical characteristics and many laboratory

indicators were observed between the carotid plaque group and the non-carotid plaque group. The age, course of the disease, NE, urea, creatinine, CysC, $\beta 2-\mathrm{MG}$, FPG, ESR in the plaque group were greater than those in the no plaque group $(P<0.05)$. In addition, the BMI, RBC, HGB, PLT, 
LY\%, ALT, AST, SUA, UUA, TP and ALB of the plaque group were lower than those in the no plaque group $(P<0.05)($ Fig. 3$)$.

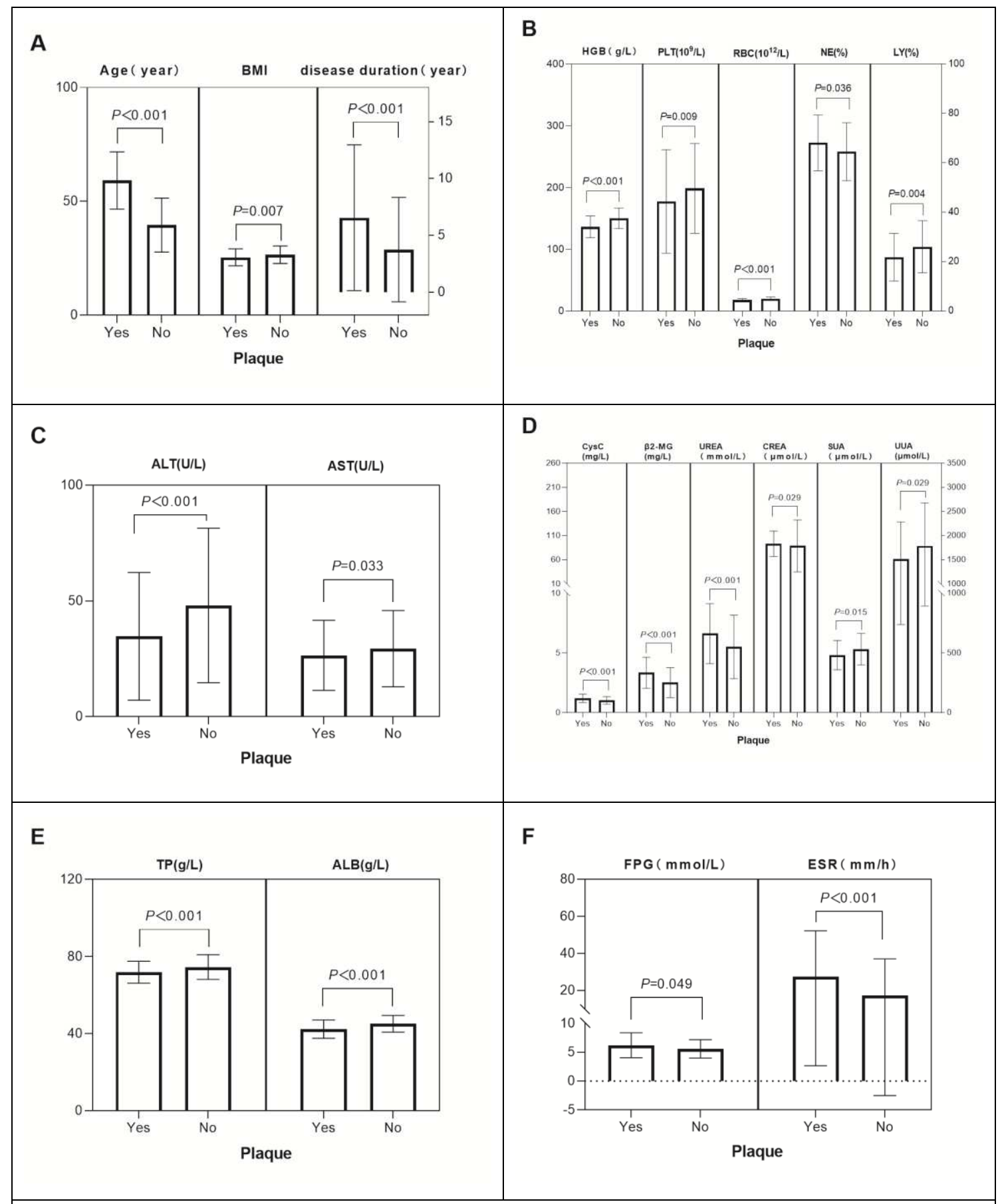

Fig. 3: Differences in general clinical characteristics and laboratory indicators between the plaque group and the non-plaque group (only show indicators with statistical differences).

A: Age and disease duration of the plaque group were higher than those of the non-plaque group, and BMI was lower than that of the non-plaque group $(P<0.001,<0.001,0.007$, respectively). B: RBC, HGB, PLT, LY\% in the plaque group were all lower than those in the non-plaque group, and NE was greater than that in the non-plaque group $(P<0.001,<0.001,0.009,0.004,0.036$, respectively). C: ALT and AST in the plaque group were lower than those in the non-plaque group $(P<0.001,0.033$, respectively). D: UREA, CREA, CysC and 
$\beta 2-\mathrm{MG}$ in the plaque group were all higher than those in the non-plaque group, and SUA and UUA were lower than those in the non-plaque group $(P<0.001,0.029,<0.001,<0.001,0.015,0.029$, respectively). E: TP and ALB in the plaque group were lower than those in the non-plaque group (all $P<0.001$ ). F: FPG and ESR in the plaque group were higher than those in the non-plaque group $(P=0.049,<0.001$, respectively).

The CCARI was positively correlated with PLT, TBIL, DBIL, and SUA $(P<0.05)$. The ICARI was positively correlated with age and DBIL $(P<0.05)$ and negatively correlated with WBC, TC, LDL-C, and ApoB $(P<0.05)$ (Table 4).

\section{Logistic regression analysis}

In this study, CCAIMT values of $>75 \%$ quantile $(0.0858 \mathrm{~cm})$ were defined as positive. The indicators that were correlated with the CCAIMT from the spearman correlation analysis, combined with hypertension, diabetes, the presence or absence of tophi, BMI, LDL-C, HDL-C, and other indicators that had an impact on the CCAIMT were included in the regression model, and multivariate binary logistic regression analysis was performed. The model was tested using the Hosmer-Lemeshow test and showed a good fit $(P=0.883)$. The results showed that age, course of the disease, LDL-C, LY\%, and SUA of patients with gout had statistically significant effects on the CCAIMT. Among them, the risk of having a CCAIMT of more than $0.0858 \mathrm{~cm}$ in patients with gout increased with age (OR, 1.136; 95\% CI, 1.073-1.202; $P<0.001)$. The course of the disease increased the risk of having a CCAIMT of more than $0.0858 \mathrm{~cm}$ in patients with gout $(\mathrm{OR}, 1.236 ; 95 \% \mathrm{CI}, 1.084-1.410 ; P=0.002)$. The LDL-C level increased the risk of having a CCAIMT of more than $0.0858 \mathrm{~cm}$ in patients with gout $(\mathrm{OR}, 6.982 ; 95 \%$ CI, $2.460-19.816 ; P<$ 0.001), whereas LY\% (OR, 0.842; 95\% CI, 0.723-0.980; $P=0.026)$ and SUA (OR, 0.994; 95\% CI, 0.988-0.999; $P=0.018$ ) reduced that risk. (Fig. 4-A).

A plaque in a neck vessel was considered positive. The indicators related to plaque, combined with hypertension, diabetes, the presence or absence of tophi, BMI, LDL-C, and HDL-C, were 
included in the regression model, and a multivariate binary logistic regression analysis was performed. The model was tested using the Hosmer-Lemeshow and showed a good fit $(P=0.972)$. The results showed that age increased the risk of cervical plaque in patients with gout (OR, 1.137; 95\% CI, 1.071-1.207; $P<0.001)$, whereas SUA reduced that risk (OR, 0.992; 95\% CI, 0.986-0.998; $P=0.015)$ (Fig. 4-B).

A CCARI of $\geq 0.700$ was defined as positive. The indicators correlated with the CCARI in the Spearman correlation analysis, combined with hypertension, diabetes, the presence or absence of tophi, age, course of the disease, and BMI, were included in the regression model, and a multivariate binary logistic regression analysis was performed. The model was tested using the Hosmer-Lemeshow test and showed a good fit $(P=0.684)$. The effects of secondary hypertension, PLT, and SUA on the CCARI were statistically significant. Patients with gout with secondary hypertension had a higher risk of having a CCARI of $\geq 0.700$ than those without hypertension $(\mathrm{OR}, 3.496 ; 95 \%$ CI, 1.442-8.479; $P=0.006)$. Both PLT (OR, 1.005; 95\% CI, 1.001-1.010; $P=$ $0.023)$ and SUA $(\mathrm{OR}, 1.003 ; 95 \% \mathrm{CI}, 1.000-1.005 ; P=0.028)$ increased the risk of having a CCARI of $\geq 0.700$ in patients with gout (Fig. 4-C).

An ICARI of $\geq 0.700$ was defined as positive. The indicators that were correlated with the ICARI in the Spearman correlation analysis, combined with the presence or absence of hypertension, diabetes, tophi, age, course of the disease, and BMI, were incorporated into the regression model, and a multivariate binary logistic regression analysis was performed. However, no variables had a statistically significant impact on the ICARI $(P>0.05)$. 


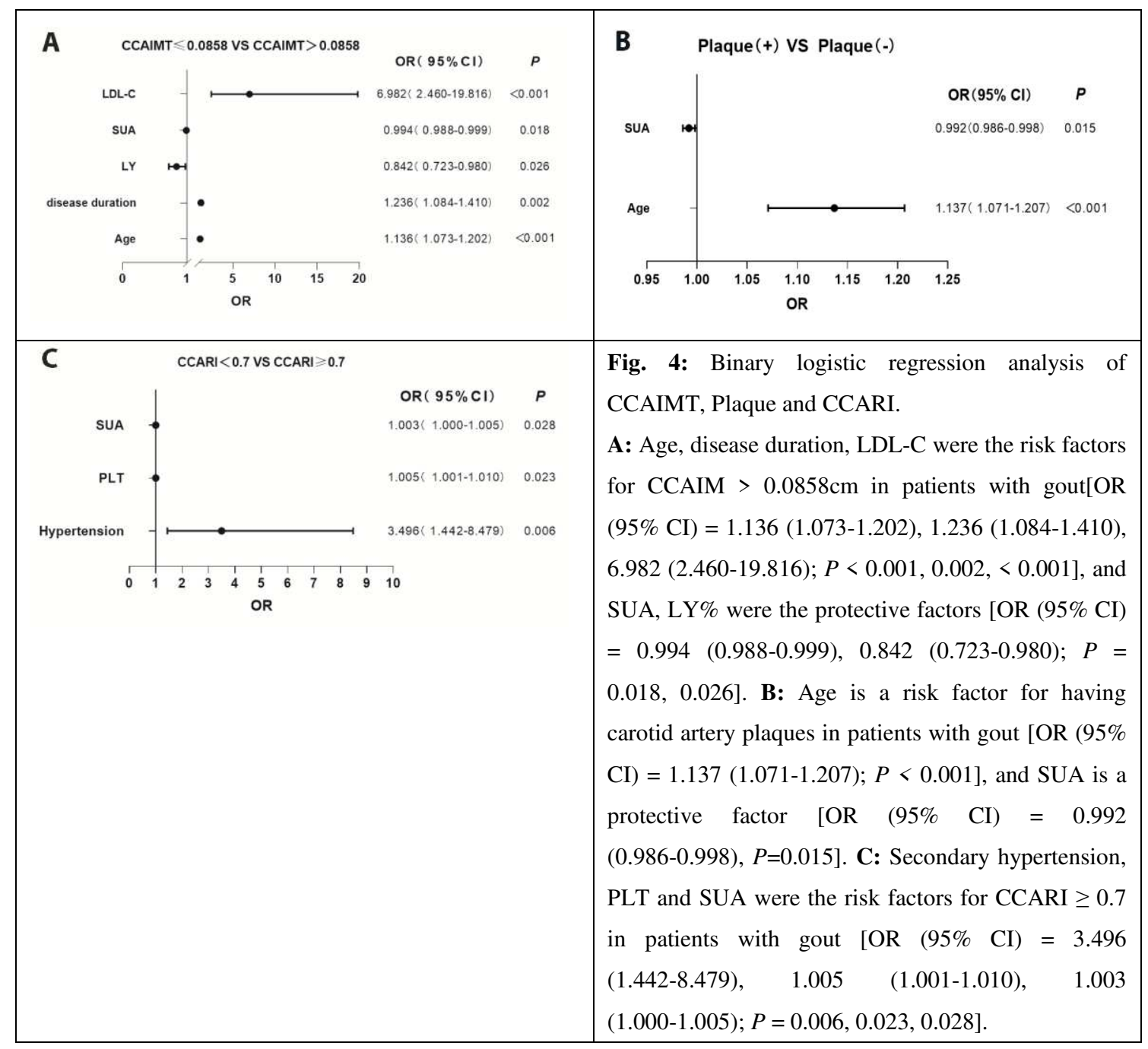

\section{Discussion}

This study found that the carotid ultrasound indexes of patients with primary gout and the CCAIMT of gout patients with different SUA levels were closely related to their age, course of the disease, blood routine indexes, renal function, lipid metabolism, and other indexes.

\section{CCAIMT and Plaque}

1.1 This study found that in patients with primary gout, SUA, and LY\% reduced the risk of carotid artery diseases. However, some studies have shown $[4,13,14,19]$ that the CIMT is not associated with SUA. Panagiota Drivelegka et al. [5] have not shown a significant correlation between the SUA quartile and CIMT or carotid plaque, regardless of gender. In addition, Mariano Andrés [16] 
et al. have found that the course of gout and the presence of tophi were not significantly related to the increase in the CIMT and plaque formation. However, José M. A. Wijnands, Arrigo F.G. Cicero, and Mingzhu Ma et al. [9-11] have shown that SUA was significantly related to the CIMT. Furthermore, a study based on patients with metabolic syndrome by Shun-Sheng Wu et al. [12] has shown that SUA is positively correlated with the CIMT. Moreover, Rada Gancheva et al. [18] have shown that in patients with asymptomatic hyperuricemia and gout, age and gouty arthritis increased the risk of CIMT abnormality (CIMT of more than $0.90 \mathrm{~mm}$ ), and the presence of tophi was the most effective risk factor. However, asymptomatic hyperuricemia did not affect the CIMT. The results of this study showed that SUA reduced the risk of having a CCAIMT of more than $0.0858 \mathrm{~cm}$ and carotid plaque formation in patients with gout, which was different from the results of previous studies. The study by Maxwell SRJ et al. [20] has shown that increasing the uric acid levels might be beneficial to patients with cardiovascular disease because of the antioxidant property of uric acid. Philip K. Tan et al. [21] have found that primates have increased their affinity for uric acid during the evolution process and, thus, inferred that maintaining a high level of SUA might provide an advantage to our primate ancestors. In addition, a multicenter study in Japan by Atsushi Tanaka et al. [22] has shown that compared with non-drug treatment, treatment with febuxostat, a uric acid-lowering agent, of patients with asymptomatic hyperuricemia for 24 months did not delay the progression of carotid atherosclerosis. In this study, SUA in patients with primary gout showed a negative effect on the CCAIMT, which might be related to its antioxidant effect. This put forward new thinking on the standard of uric acid-lowering treatment for patients with gout, especially for elderly patients, whether the standard of uric acid-lowering treatment should be appropriately relaxed to improve the protective effect of uric acid on blood vessels. 
However, the sample size needs to be further expanded in the future to confirm this conclusion.

In addition, the results of this study showed that LY\% was a protective factor for CCAIMT, and LY\% could reduce the risk of CCAIMT thickening. Studies have shown that macrophages and LY\% play a key role in the development of atherosclerosis and can provide signals for the potential pro-inflammatory background of atherosclerosis [23]. The results of this study were inconsistent with the aforementioned pathological process. However, in the study by Mucahid Yilmaz et al. [24], the neutrophil/lymphocyte ratio (NLR) was an index reflecting high neutrophil levels (indicating inflammation) and lymphopenia induced by physiological stress. In addition, the NLR level of the coronary artery disease group was higher than that of the normal coronary artery group, indicating that low LY\% levels were an index that was not conducive to the coronary artery. In this study, the increase in LY\% in patients with gout helped reduce the probability of CCAIMT lesions, which was consistent with the study by Mucahid Yilmaz. However, few studies have focused on the relationship between LY\% and arteriosclerosis in patients with primary gout. This study may provide a new direction for future research, hoping to provide a more comprehensive reference index for preventing and treating clinical gout vascular complications.

Age and LDL-C increasing the risk of atherosclerosis has been confirmed by many studies $[23,25]$, and the results of this study also confirmed this. In addition, the results of this study showed that the course of gout increased the risk of CCAIMT thickening, which was inconsistent with the results of the study by Mariano Andrés [16]. The reason might be that the course of gout in this study was positively correlated with age (age and disease course were Spearman-related: $\mathrm{R}$ $=0.312 ; P<0.001)$.

\subsection{Different SUA levels and CCAIMT}


The study by Shinji Kishimoto et al. [26] has proven that Hct, HGB, and RBC were related to the brachial artery IMT in male patients, and the increase in Hct, HGB, and RBC content might reduce the risk of cardiovascular diseases. The correlation analysis between different SUA levels and the CCAIMT in this study showed that the RBC count in the patients with gout in the Q1-Q4 groups were negatively correlated with the CCAIMT, and the HGB level of the patients with gout in the Q2-Q4 groups were negatively correlated with the CCAIMT. We could infer that within the normal range, relatively high RBC and HGB levels were beneficial to the CCAIMT in patients with gout with different SUA levels.

In this study, the CysC of the patients with gout in the Q1-Q4 groups were positively correlated with the CCAIMT, and the $\beta-2 \mathrm{MG}$ of the patients with gout in the Q2-Q4 groups were positively correlated with the CCAIMT. Regarding CysC, a study by Chiu-Shong Liu et al. [27] has shown that $\mathrm{CysC}$ and CIMT have a strong linear positive correlation in Taiwanese residents. Furthermore, the level of CysC increased, and CIMT thickened. The studies by Jing Ren, Abdullah Demirbaş, Caroline Fuchs Einwoegerer, etc. [28-30] have shown that CysC is an important marker of atherosclerosis and is significantly related to cardiovascular events. However, some studies have reached the opposite conclusion. The results of a Mendelian randomized study by Sander W. van der Laan [31] did not support the causal role of CysC in the etiology of cardiovascular diseases. Regarding $\beta-2 \mathrm{MG}$, the studies by Andrew M. Wilson and Michel M. Joosten $[32,33]$ have shown that $\beta-2 \mathrm{MG}$ is related to the increased risk of peripheral vascular diseases and their severity. Moreover, Copenhagen Lupus et al. [34] have shown that the increase in $\beta-2 \mathrm{MG}$ in patients with systemic lupus erythematosus was significantly associated with carotid plaque and coronary calcification. We can speculate that in patients with gout with different levels 
of SUA, CysC and $\beta-2 M G$ are unfavorable for the CCAIMT. Perhaps, reducing CysC and $\beta-2 \mathrm{MG}$ to protect patients with primary gout from cervical vascular damage is clinically possible.

A study by Shigeru Hayashi et al. [35] has shown that the CIMT is positively correlated with age, creatinine, BUN, and C-reactive protein and negatively correlated with TP and ALB in patients with atherosclerosis, which was consistent with the results of this study. It is worth mentioning that creatinine is not associated with the overall CCAIMT in this study, whereas it is positively correlated with the CCAIMT in the Q3 and Q4 groups (SUA levels of more than 510 $\mathrm{mmol} / \mathrm{L}$ ). It might be inferred from this that patients with gout with high SUA levels should be alert to creatinine levels to better prevent vascular complications.

\section{CCARI}

A series of studies involving patients with asymptomatic hyperuricemia and gout by Rada N.

Gancheva $[17,18]$ have shown that hypertension significantly increased the risk of CCARI abnormalities, the presence of tophi significantly increased the possibility of having a CCARI of $\geq$ 0.7 in patients with gout, and gout with tophi and hyperuricemia had no significant effect on the risk of CCARI abnormalities. The results of this study showed that both SUA and hypertension were risk factors for having a CCARI of $\geq 0.7$, which is consistent with the results of previous studies. This study suggests that treating gout early is essential.

Several studies have shown that [36-41], in addition to hemostasis, platelets are also involved in the process of atherosclerosis. Constantinos Bakogiannis et al. [40] have found that platelets could release various chemokines and recruit and activate immune cells during vascular injuries, such as monocytes, neutrophils, and $\mathrm{T}$ lymphocytes, thereby playing a role in vascular inflammation and atherosclerosis. Studies by Norbert Gerdes, Tessa J. Barrett et al. [38, 41] have 
also shown that platelets promote atherosclerosis. This study found that PLT was a risk factor for having a CCARI of $\geq 0.7$ in patients with gout, which was consistent with the role of PLT in previous studies. The results of this study suggest that we can indirectly predict the impact of platelets on the CCARI by detecting their changes during the diagnosis and treatment of gout.

\section{ICARI}

The results of study by the José Alexandre Addeo Cipolli [19] et al. have shown that SUA is not significantly correlated with the CIMT in men and women. After adjusting for potential confounding factors, regression analysis has shown that the ICARI was independently associated with SUA and hyperuricemia in women. The results of the regression analysis in this study did not find risk factors for ICARI in patients with gout, but the proportion of women in this study was small, which might be the reason for the inconsistency with the results of this study and previous studies.

\section{Conclusion}

In summary, this study found that the CCAIMT in patients with primary gout with different SUA levels was closely related to blood routine indexes, renal function, lipid metabolism, and other indicators. Especially for patients with gout with high SUA levels, the CCAIMT was positively correlated with several renal function-related indicators, among which creatinine might be an observed index of carotid artery disease in patients with gout with high SUA level. In patients with primary gout, age, course of the disease, and LDL-C might be risk factors for carotid intima-media diseases, suggesting that we should pay attention to early diagnosis and treatment of gout to prevent carotid intima-media-related lesions. Meanwhile, SUA and LY\% are possible protective factors for carotid arterial intima-media lesions in patients with primary gout. The study 
can be expanded in the future to provide a new reference for the standard of uric acid treatment for vascular protection in patients with primary gout. The limitations of this study were that the sample size was small and the proportion of women was low. In the future, the sample size could be expanded and the gender ratio could be further studied to confirm the results of this study.

\section{Acknowledgements}

We thank PhD. Jian Liu and Prof. Jingguo Zhou for insightful suggestions.

\section{Authors' contributions}

W-TD conceived and designed the work. W-TD and JH contributed to analyses of imaging data, prepared the figures, and provided technical support. D-Ll, HL and XX contributed to data collection and interpretation of data. JH drafted the manuscript. W-TD and and JL critically revised the manuscript for important intellectual content. All authors read and approved the final manuscript.

\section{Funding}

This work was supported by the National Natural Science Foundation of China (No. 81603441), the Basic Application Research Project of Sichuan Science and Technology Department (No. 2017JY0297), Key Projects of Health Commission of Sichuan Province (No. 18ZD041), the Innovative Team Building Project of Chengdu Medical College (No. CYTD18-03), the School Cooperation Project of Nanchong (No. NSMC20170452), and the Key Projects of The First Affiliated Hospital of Chengdu Medical College (No. CYFY2019ZD01), Project of Sichuan Provincial Administration of traditional Chinese Medicine (No. 2020JC0023). Scientific research project of Sichuan Provincial Health Commission (key research project, No. 19ZD004).

\section{Ethics approval and consent to participate}


All participants provided informed written consent. This study was approved by the Medical Ethics Committee of the Affiliated Hospital of Chengdu Medical College (2018CYFYIRB-BA-02).

\section{Consent for publication}

Not applicable.

\section{Competing interests}

The authors declare that they have no competing interests.

\section{Reference}

[1]. Richette P, Doherty M, Pascual E, Barskova V, Becce F, Castaneda J, et al. 2018 updated European League Against Rheumatism evidence-based recommendations for the diagnosis of gout. Ann Rheum Dis. 2020; 79(1):31-8

[2]. Neogi T, Jansen TL, Dalbeth N, Fransen J, Schumacher HR, Berendsen D, et al. 2015 Gout classification criteria: an American College of Rheumatology/European League Against Rheumatism collaborative initiative. Ann Rheum Dis. 2015; 74(10):1789-98.

[3]. Dehlin M, Jacobsson L, Roddy E. Global epidemiology of gout: prevalence, incidence, treatment patterns and risk factors. Nat Rev Rheumatol. 2020; 16(7):380-390.

[4]. Borghi C, Agabiti-Rosei E, Johnson RJ, Kielstein JT, Lurbe E, Mancia G, et al. Hyperuricaemia and gout in cardiovascular, metabolic and kidney disease. Eur J Intern Med. 2020; 80:1-11.

[5]. Drivelegka P, Forsblad-d'Elia H, Angerås O, Bergström G, Schmidt C, Jacobsson LTH, et al. Association between serum level of urate and subclinical atherosclerosis: results from the SCAPIS Pilot. Arthritis Res Ther. 2020; 22(1):37.

[6]. Roman MJ, Naqvi TZ, Gardin JM, Gerhard-Herman M, Jaff M, Mohler E. Clinical application of noninvasive 
vascular ultrasound in cardiovascular risk stratification: a report from the American Society of Echocardiography and the Society of Vascular Medicine and Biology. J Am Soc Echocardiogr. 2006; 19(8):943-54.

[7]. Chambless LE, Folsom AR, Davis V, Sharrett R, Heiss G, Sorlie P, et al. Risk factors for progression of common carotid atherosclerosis: the Atherosclerosis Risk in Communities Study, 1987-1998. Am J Epidemiol. $2002 ; 155(1): 38-47$.

[8]. Staub D, Meyerhans A, Bundi B, Schmid HP, Frauchiger B. Prediction of cardiovascular morbidity and mortality: comparison of the internal carotid artery resistive index with the common carotid artery intima-media thickness. Stroke. 2006; 37(3):800-5.

[9]. Cicero AF, Salvi P, D'Addato S, Rosticci M, Borghi C; Brisighella Heart Study group. Association between serum uric acid, hypertension, vascular stiffness and subclinical atherosclerosis: data from the Brisighella Heart Study. J Hypertens. 2014; 32(1):57-64.

[10]. Wijnands JM, Boonen A, Dagnelie PC, van Greevenbroek MM, van der Kallen CJ, Ferreira I, et al. The cross-sectional association between uric acid and atherosclerosis and the role of low-grade inflammation: the CODAM study. Rheumatology (Oxford). 2014; 53(11):2053-62.

[11]. Ma M, Wang L, Huang W, Zhong X, Li L, Wang H, et al. Meta-analysis of the correlation between serum uric acid level and carotid intima-media thickness. PLoS One. 2021; 16(2):e0246416.

[12]. Wu SS, Kor CT, Chen TY, Liu KH, Shih KL, Su WW, et al. Relationships between Serum Uric Acid, Malondialdehyde Levels, and Carotid Intima-Media Thickness in the Patients with Metabolic Syndrome. Oxid Med Cell Longev. 2019; 2019:6859757.

[13]. Bae JS, Shin DH, Park PS, Choi BY, Kim MK, Shin M, et al. The impact of serum uric acid level on arterial stiffness and carotid atherosclerosis: The Korean Multi-Rural Communities Cohort study. Atherosclerosis. 
2013; 231(1):145-51.

[14]. Oikonen M, Wendelin-Saarenhovi M, Lyytikäinen L, Siitonen N, Loo B, Jula A, et al. Associations between serum uric acid and markers of subclinical atherosclerosis in young adults. The cardiovascular risk in Young Finns study. Atherosclerosis. 2012; 223(2):497-503.

[15]. Touboul PJ, Hennerici MG, Meairs S, Adams H, Amarenco P, Bornstein N, et al. Mannheim carotid intima-media thickness and plaque consensus (2004-2006-2011). An update on behalf of the advisory board of the 3rd, 4th and 5th watching the risk symposia, at the 13th, 15th and 20th European Stroke Conferences, Mannheim, Germany, 2004, Brussels, Belgium, 2006, and Hamburg, Germany, 2011. Cerebrovasc Dis. 2012; 34(4):290-6.

[16]. Andrés M, Bernal JA, Sivera F, Quilis N, Carmona L, Vela P, et al. Cardiovascular risk of patients with gout seen at rheumatology clinics following a structured assessment. Ann Rheum Dis. 2017; 76(7):1263-8.

[17]. Gancheva RN, Kundurdjiev AI, Ivanova MG, Kundurzhiev TG, Rashkov RK, Kolarov ZG. Ultrasonographic measurement of carotid artery resistive index and diastolic function of the heart in gout patients. Rheumatol Int. 2015; 35(8):1369-75.

[18]. Gancheva R, Kundurdjiev A, Ivanova M, Kundurzhiev T, Kolarov Z. Evaluation of cardiovascular risk in stages of gout by a complex multimodal ultrasonography. Rheumatol Int. 2017; 37(1):121-30.

[19]. Cipolli JA, Ferreira-Sae MC, Martins RP, Pio-Magalhães JA, Bellinazzi VR, Matos-Souza JR, et al. Relationship between serum uric acid and internal carotid resistive index in hypertensive women: a cross-sectional study. BMC Cardiovasc Disord. 2012; 12:52.

[20].Maxwell SR, Thomason H, Sandler D, Leguen C, Baxter MA, Thorpe GH, et al. Antioxidant status in patients with uncomplicated insulin-dependent and non-insulin-dependent diabetes mellitus. Eur J Clin Invest. 1997; 27(6):484-90. 
[21].Tan PK, Farrar JE, Gaucher EA, Miner JN. Coevolution of URAT1 and Uricase during Primate Evolution: Implications for Serum Urate Homeostasis and Gout. Mol Biol Evol. 2016; 33(9):2193-200.

[22]. Tanaka A, Taguchi I, Teragawa H, Ishizaka N, Kanzaki Y, Tomiyama H, et al. Febuxostat does not delay progression of carotid atherosclerosis in patients with asymptomatic hyperuricemia: A randomized, controlled trial. PLoS Med. 2020; 17(4):e1003095.

[23].Ferrari D, la Sala A, Milani D, Celeghini C, Casciano F. Purinergic Signaling in Controlling Macrophage and T Cell Functions During Atherosclerosis Development. Front Immunol. 2021; 11:617804.

[24].Yılmaz M, Korkmaz H, Bilen MN, Uku Ö, Kurtoğlu E. Could neutrophil/lymphocyte ratio be an indicator of coronary artery disease, coronary artery ectasia and coronary slow flow? J Int Med Res. 2016; 44(6):1443-53.

[25].Xing L, Li R, Zhang S, Li D, Dong B, Zhou H, et al. High Burden of Carotid Atherosclerosis in Rural Northeast China: A Population-Based Study. Front Neurol. 2021; 12:597992.

[26].Kishimoto S, Maruhashi T, Kajikawa M, Matsui S, Hashimoto H, Takaeko Y, et al. Hematocrit, hemoglobin and red blood cells are associated with vascular function and vascular structure in men. Sci Rep. 2020; 10(1):11467.

[27].Liu CS, Li CI, Guo YC, Lin CH, Lin WY, Liu CH, et al. Independent associations of urinary albumin-to-creatinine ratio and serum cystatin $\mathrm{C}$ with carotid intima-media thickness in community-living Taiwanese adults. BMC Nephrol. 2020; 21(1):454.

[28].Einwoegerer CF, Domingueti CP. Association Between Increased Levels of Cystatin C and the Development of Cardiovascular Events or Mortality: A Systematic Review and Meta-Analysis. Arq Bras Cardiol. 2018; 111(6):796-807.

[29].Larsen TR, Gerke O, Diederichsen ACP, Lambrechtsen J, Steffensen FH, Sand NP, et al. Lack of association between cystatin C and different coronary atherosclerotic manifestations. Scand J Clin Lab Invest. 2017 ; 
77(8):574-581.

[30].Ren J, Dong X, Nao J. Serum cystatin C is associated with carotid atherosclerosis in patients with acute ischemic stroke. Neurol Sci. 2020; 41(10):2793-800.

[31].van der Laan SW, Fall T, Soumaré A, Teumer A, Sedaghat S, Baumert J, et al. Cystatin C and Cardiovascular Disease: A Mendelian Randomization Study. J Am Coll Cardiol. 2016; 68(9):934-45.

[32].Wilson AM, Kimura E, Harada RK, Nair N, Narasimhan B, Meng XY, et al. Beta2-microglobulin as a biomarker in peripheral arterial disease: proteomic profiling and clinical studies. Circulation. 2007; 116(12):1396-403.

[33].Joosten MM, Pai JK, Bertoia ML, Gansevoort RT, Bakker SJ, Cooke JP, et al. $\beta 2$-microglobulin, cystatin C, and creatinine and risk of symptomatic peripheral artery disease. J Am Heart Assoc. 2014; 3(4):e000803.

[34].Leffers HCB, Hermansen ML, Sandholt B, Fuchs A, Sillesen H, Køber L, et al. Plasma levels of $\beta 2$-microglobulin are associated with atherosclerosis in patients with systemic lupus erythematosus: a cross-sectional cohort study. Lupus. 2018; 27(9):1517-23.

[35].Hayashi S. Significance of plasma D-dimer in relation to the severity of atherosclerosis among patients evaluated by non-invasive indices of cardio-ankle vascular index and carotid intima-media thickness. Int $\mathrm{J}$ Hematol. 2010; 92(1):76-82.

[36]. Sreeramkumar V, Adrover JM, Ballesteros I, Cuartero MI, Rossaint J, Bilbao I, et al. Neutrophils scan for activated platelets to initiate inflammation. Science. 2014; 346(6214):1234-8.

[37]. Ed Rainger G, Chimen M, Harrison MJ, Yates CM, Harrison P, Watson SP, et al. The role of platelets in the recruitment of leukocytes during vascular disease. Platelets. 2015; 26(6):507-20.

[38]. Gerdes N, Seijkens T, Lievens D, Kuijpers MJ, Winkels H, Projahn D, et al. Platelet CD40 Exacerbates Atherosclerosis by Transcellular Activation of Endothelial Cells and Leukocytes. Arterioscler Thromb Vasc 
Biol. 2016; 36(3):482-90.

[39]. Marx C, Novotny J, Salbeck D, Zellner KR, Nicolai L, Pekayvaz K, et al. Eosinophil-platelet interactions promote atherosclerosis and stabilize thrombosis with eosinophil extracellular traps. Blood. 2019; 134(21):1859-72.

[40].Bakogiannis C, Sachse M, Stamatelopoulos K, Stellos K. Platelet-derived chemokines in inflammation and atherosclerosis. Cytokine. 2019; 122:154157.

[41].Barrett TJ, Schlegel M, Zhou F, Gorenchtein M, Bolstorff J, Moore KJ, et al. Platelet regulation of myeloid suppressor of cytokine signaling 3 accelerates atherosclerosis. Sci Transl Med. 2019; 11(517):eaax0481. 
Figures

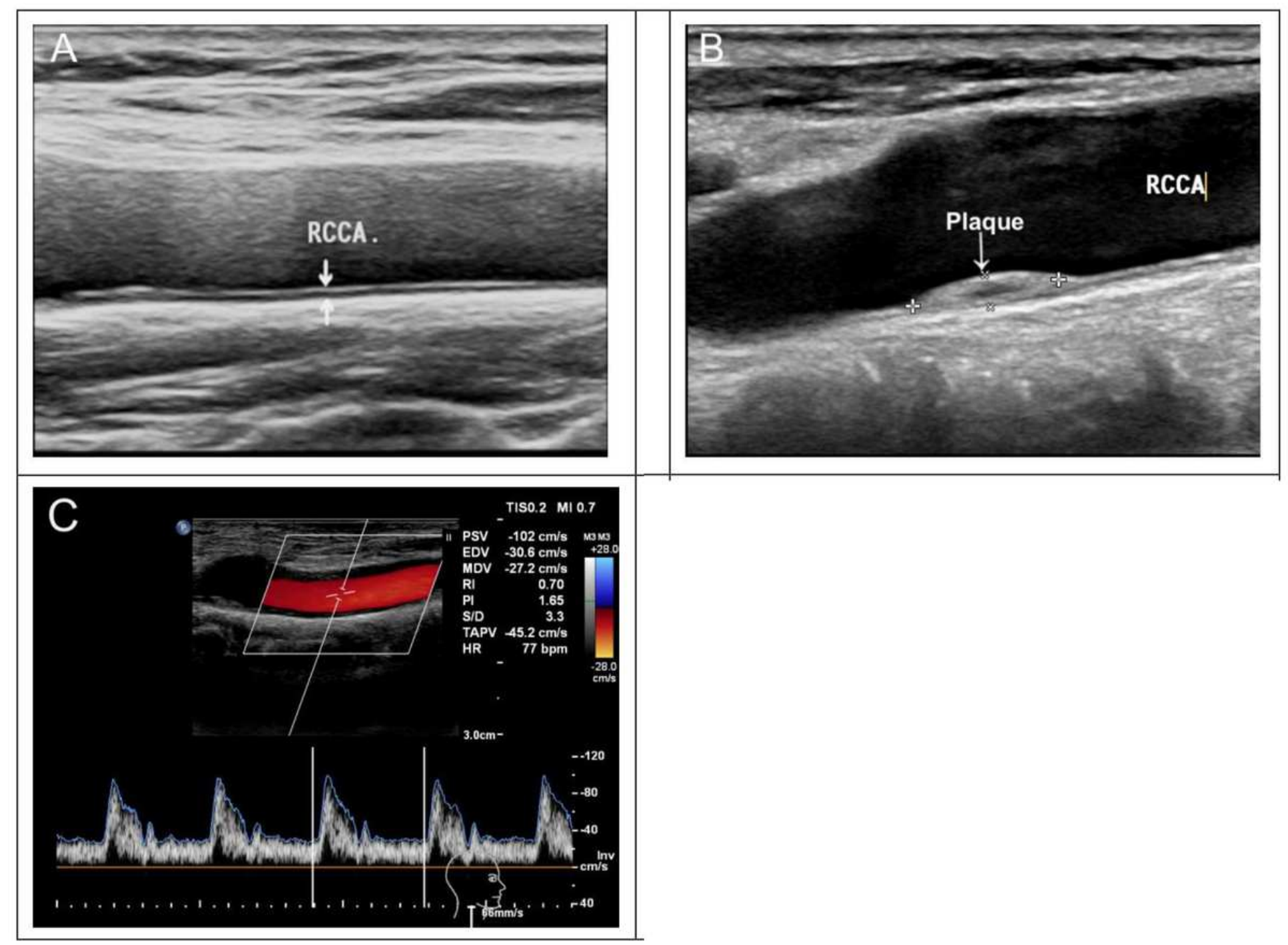

Figure 1

Please see the Manuscript PDF file for the complete figure caption 


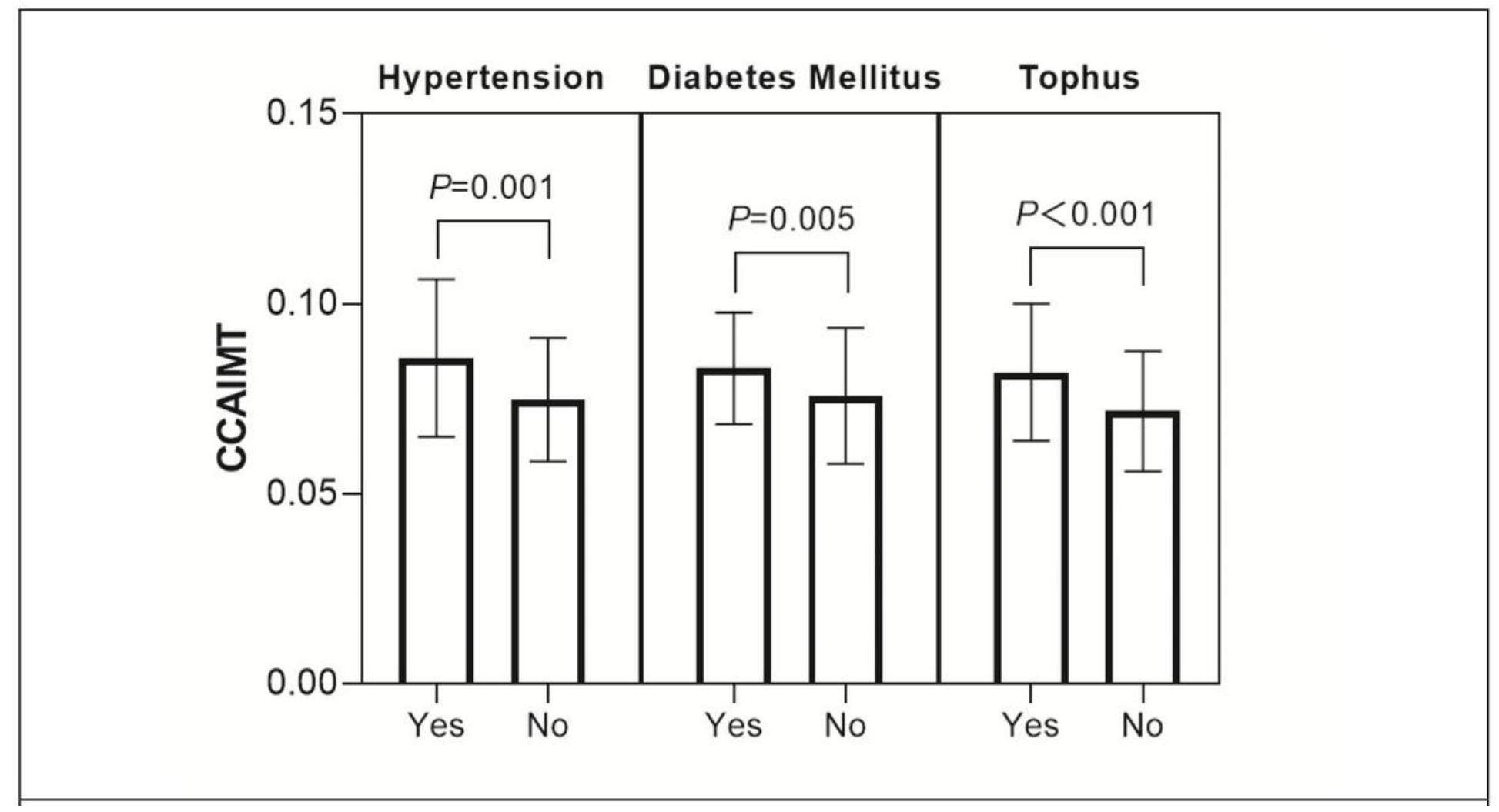

Figure 2

Please see the Manuscript PDF file for the complete figure caption 


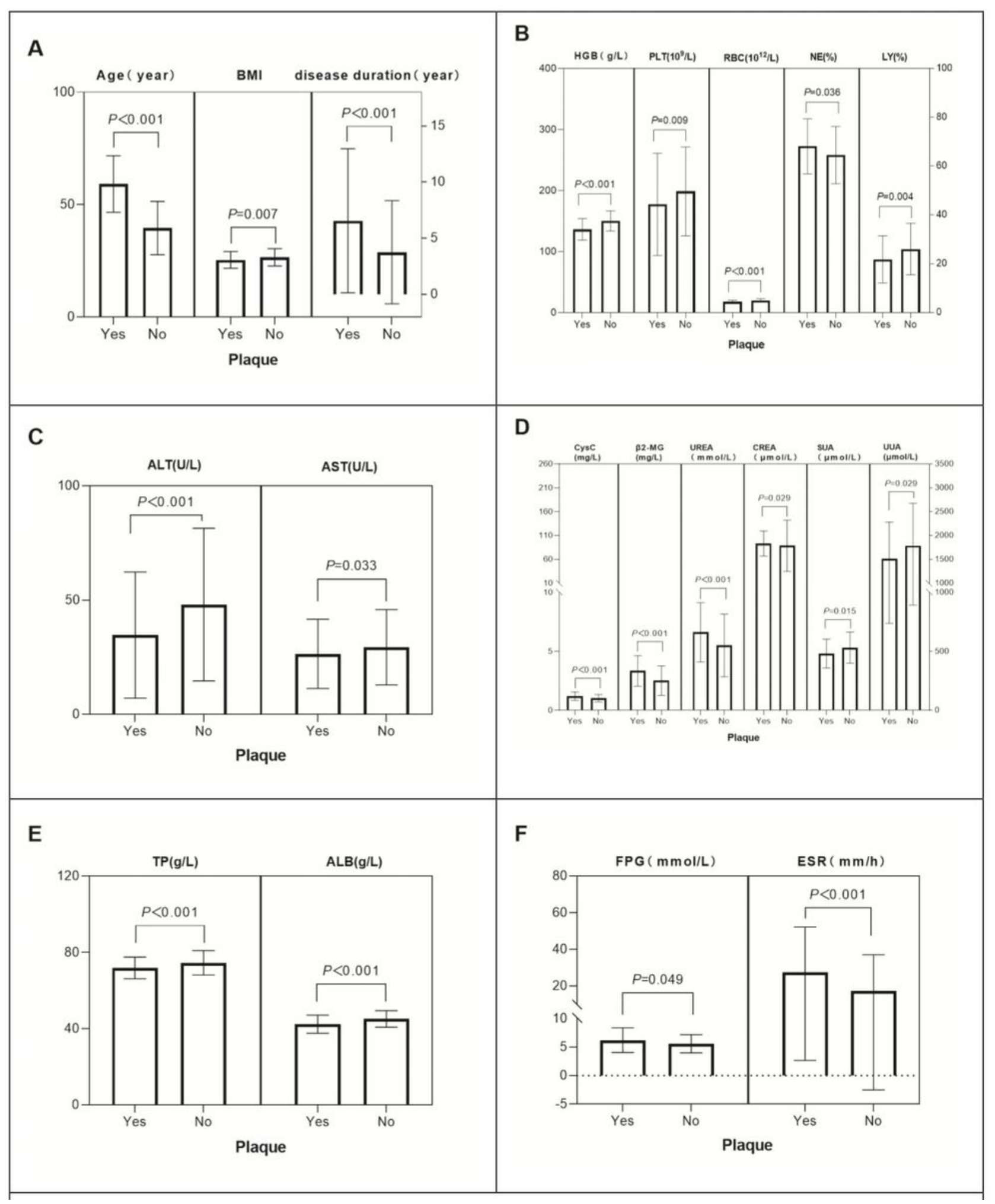

Figure 3

Please see the Manuscript PDF file for the complete figure caption 


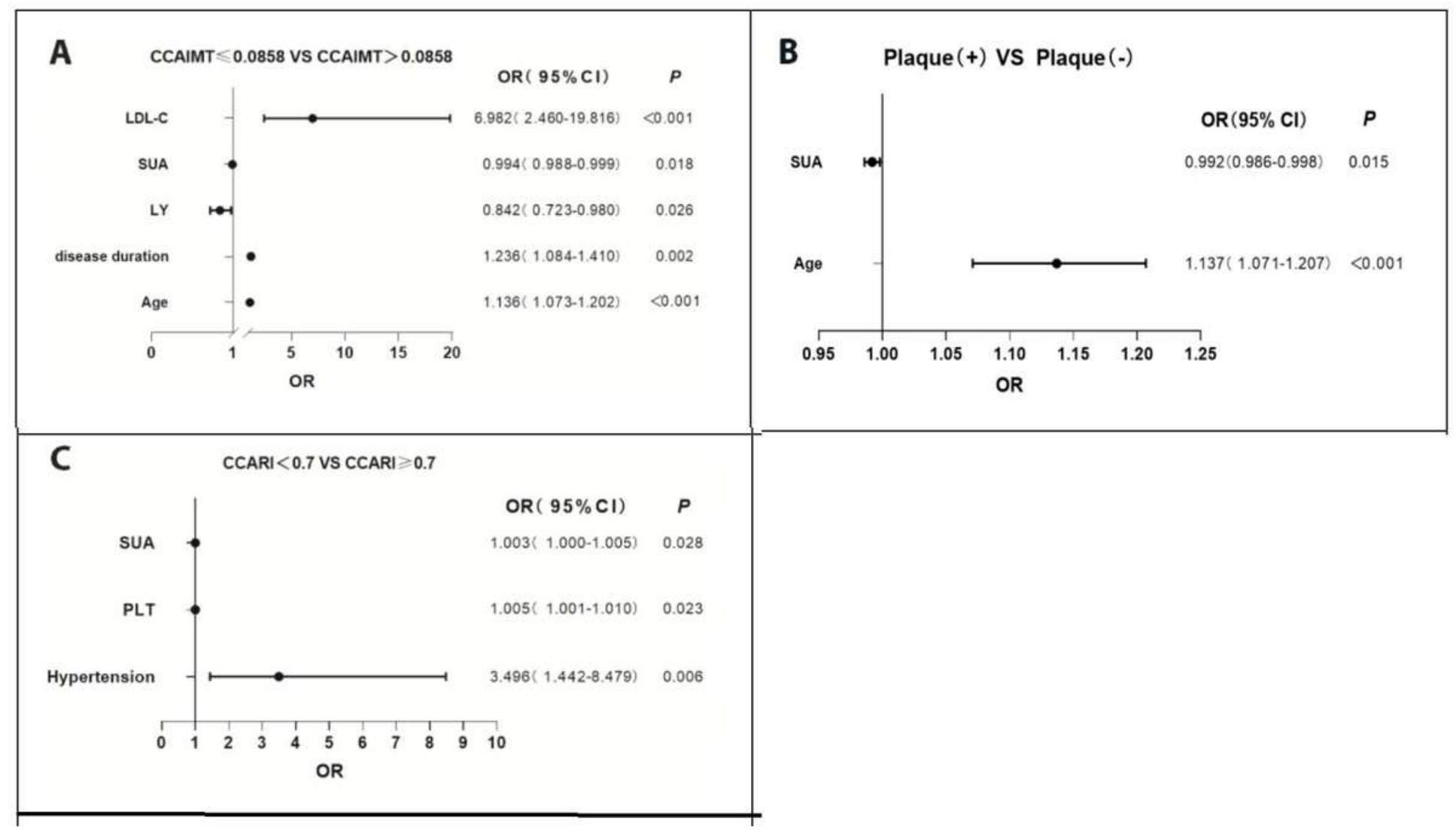

Figure 4

Please see the Manuscript PDF file for the complete figure caption 